\title{
Energy Efficient Lighting System in Gaza Strip Buildings
}

\author{
Hatem Elaydi*, Zaki Al Qaraa \\ Electrical Engineering Department, Islamic University of Gaza, Gaza, Palestine \\ *Corresponding author: helaydi@iugaza.edu.ps
}

Received October 11, 2013; Revised February 09, 2014; Accepted February 11, 2014

\begin{abstract}
This study aimed at identifying design features that can be incorporated in buildings to make them energy efficient. Energy audit and analysis is necessary to increase building energy efficiency. Three methods to increase energy efficiency were selected based on installation cost: the first method of low cost is replacing T-8 fluorescent lamps (FL) and Metal Halide lamps (ML) with T-5 and High Pressure Sodium (HPS) respectively, the second method of medium cost is replacing all FL and ML lamp with Light Emitting Diode (LED), and the third method of high cost is the automation system for room lighting based on fuzzy logic controller at some places in the building. Energy audit and analysis was performed on Irada building at Islamic University of Gaza (IUG). Good energy saving were achieved using low cost, medium cost, and high cost methods. Finally, this provided much needed power saving to be reused in Gaza Strip.
\end{abstract}

Keywords: lighting, energy, fluorescent, light emitting diode, electrical defect

Cite This Article: Hatem Elaydi, and Zaki Al Qaraa, "Energy Efficient Lighting System in Gaza Strip Buildings.” American Journal of Electrical and Electronic Engineering, vol. 2, no. 2 (2014): 57-61. doi: 10.12691/ajeee-2-2-5.

\section{Introduction}

According to the International Energy Agency, IEA, generated power can be increased by finding renewable power energy; moreover, saving measures can increase the available power [1]. Today, buildings worldwide account for up to $40 \%$ of total end-use energy. The US, OECD/ Europe and Russia consume most of their energy in the building sector (about 48\%) as shown in Figure 1 [2]. The consumption of electricity in Gaza Strip is distributed such as the residential buildings sector (73\%), industrial (16\%), commercial (8\%) and others (3\%).

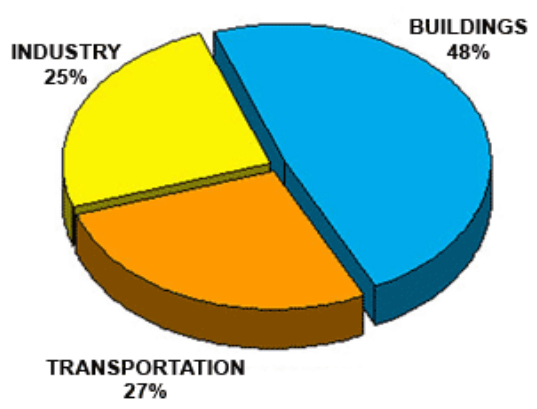

Figure 1. Global energy demand by sector in 2005

Much of the increased demand for electricity is due to the increased standard of living among the wealthier income groups. One of the major factors in the increased use of electricity by the higher income group is the use of air conditioning units which has recently become quite popular in the area. The increase of electricity use in Gaza Strip residential buildings during the summer and winter months has been caused by the growing demands for lighting system. By the year 2020, the population of Gaza will increase to around 2.13 million from an estimated 1.6 million people today [3].

The substantial population growth rate will add some 500,000 people to a living area which is restricted and is already heavily urbanized. Fundamental infrastructure in electricity, water and sanitation, municipal and social services, is struggling to keep pace with the needs of the growing population. Electricity provision needs to double every five years in order to meet the growing demands, and the expansion in new schools and health services of the overwhelmingly young population. Tens of thousands of new housing units are needed today; thus, there is a need for a new code of energy saving buildings.

Moreover, a study of the regulations in the national building code of Palestine shows that the building codes do not address the issues of energy efficiency in any building category. Architects and developers of residential buildings, too, have not considered ways in which energy use can be reduced. This will add to the current problem of continued shortage in electrical power supply where the demands keep increasing while the supply is not growing at all.

The specific problems that signify the importance of energy-efficiency in residential buildings are as follows: High-energy use of residential buildings in Gaza, growing population with new apartments and homes, and increased standards of living. That led to interrupted power supply due to power deficits and scheduled power outage. All of 
the above reasons were motives to find out solutions that would reduce the electrical deficit rate in Gaza Strip.

This paper presents several new technologies that offer solutions for the lighting system such as replacing T-8 FL by T-5 and ML by HPS, replacing all fluorescent lamps and ML lamp with LED lamps, and automation System for room lighting based on fuzzy logic control; thus, improvement of the efficiency of electricity sector in Gaza Strip can be made.

This paper is organized such as: section 2 talks about the Irada building as a case study, section 3 presents approaches and methodologies for electrical energy saving and shows the potential saving, section 4 presents the results, and section 5 concludes this paper.

\section{Case Study Building Specification}

Irada building hosts more than one hundred craftsmen; in addition to, some College of Commerce staff and trainers. The working hours are between 1600 to 1800 hours per year for an average of 6-8 hours daily with five working days weekly. The building consists of three floors: basement, ground floor and the first floor. All workshops are in the basement floor, all management offices and laboratory are in ground floor while showrooms are in first floor. Table 1 presents the annual electrical consumption of the lighting system in all floors of Irada building.

Table 1. Annual Electrical Consumption of Lighting System

\begin{tabular}{|c|c|c|c|c|}
\hline No & Lamp Type & lumen output/Watt & Wattage & Lumen \\
\hline 1 & T-8 & 75 lumen/w & $48 \mathrm{~W}$ & 3600 \\
\hline 2 & T-5 & 90 lumen/w & $32 \mathrm{~W}$ & 2880 \\
\hline
\end{tabular}

\section{Lighting System Energy Saving Methods}

\subsection{Replace T-8 FL by T-5 and ML by HPS}

T-8 and T-5 FL tubes on the market today have the typical performance and technical specifications as stated in Table II. In terms of energy consumption, a $1200 \mathrm{~mm}$ T-5 FL tube uses about 33\% less energy than a T-8 FL, but due to the lower voltage output, a T-5 FL tube would also generate less light than a T-8 FL tube.
For example, if a T-8 FL tube is replaced with a T-5 FL tube under a control situation where condition remains the same; there should be a theoretical decrease in brightness of about $20-30 \%$. This calculation could easily be verified by simply measuring the Lux levels at a specified distance [4]. With the addition of the special light enhancing reflector, the normally waste light of about 30\% beaming towards the backside of the light tray is reflected back downwards; thus, becoming useful light again [4].

The total useful light from a T-5 tube beaming downward would effectively be equal to the light from a $\mathrm{T}-8$ light tube [9]. In other word, this combination of T-5 light tube with reflector could achieve the 33\% energy saving whilst matching the brightness performance of a $\mathrm{T}$ 8 light tube, i.e. one-to-one replacement [4]. By achieving one-to-one replacement, there would be no need to add additional light tubes or trays in order to compensate for the decrease in brightness level. Alteration or additional works to the existing lighting arrangement could therefore be minimized [5].

Using the data in Table 2, we can find out the annual energy saving made by lights fitting replacement method (i.e. T5 instead T8 and HPS instead of ML) where an annual energy saving of $18363.2 \mathrm{kWh} /$ year is made after installing the new technology of high efficient LED lamps.

Table 2. T-8 VS. T-5 FL tube technical specification

\begin{tabular}{|c|c|c|c|c|c|}
\hline \multicolumn{1}{|c|}{ Table 2. T-8 VS. T-5 FL tube technical specification } \\
\cline { 3 - 6 } No. & \multirow{2}{*}{ Light type } & Q & rate & OH & Energy used \\
\cline { 3 - 6 } & & Nos & W & hr & kWh/year \\
\hline 01 & $2 \times 36$ W FL & 188 & 77.4 & 1600 & 23281.92 \\
\hline 02 & $1 \times 36$ WL & 23 & 41.4 & 1600 & 1523.52 \\
\hline 03 & $1 \times 26$ W CFL & 12 & 26 & 1600 & 499.20 \\
\hline 04 & $4 \times 18 W$ FL & 51 & 77.6 & 1600 & 6332.16 \\
\hline 05 & $1 \times 400$ W ML & 8 & 400 & 2920 & 9344.00 \\
\hline
\end{tabular}

\subsection{Replacement of LED Lamps Instead of All Fluorescent Lamps and ML Lamp}

In this method, we will replace lamps from fluorescent lamps $36 \mathrm{~W}$ by high technology LED tube lamps $18 \mathrm{~W}$ [6] in specific places such as workshops, laboratories and offices, we also replace $150 \mathrm{~W}$ HPS by $54 \mathrm{~W}$ led light [7]. Table 3 shows comparison between LED and FL tube. Expected annual energy achieved upon replacing lamps from linear fluorescent lamps T8 to high technology LED lamps is $24791.36 \mathrm{kWh} /$ year.

Table 3. Comparison between led and fl tube

\begin{tabular}{|c|c|c|c|}
\hline No & Characteristic & LED tube & Conventional Fluorescent tube \\
\hline 1 & Power consumption (tube only) & $18 \mathrm{~W}$ & $36 \mathrm{~W}$ \\
\hline 2 & Life span & 40,000 hours & 8,000 hours \\
\hline 3 & Radiation & CE compliant, no UV or IR & UV, IR \\
\hline \multirow{2}{*}{4} & \multirow{2}{*}{ Toxic contents } & \multirow{2}{*}{ RoHS compliant } & Toxic phosphor powders \\
\hline & & & Mercury (Hg), Lead (Pb) \\
\hline 5 & $\mathrm{CO}_{2}$ emission & Low & High \\
\hline 6 & Heat damage & No & High \\
\hline \multirow{2}{*}{7} & \multirow{2}{*}{ Durable } & Unbreakable & \multirow{2}{*}{ Fragile Glass } \\
\hline & & Aluminum Housing and Poly Carbonate Cover & \\
\hline 8 & Burn out failure & No & Yes \\
\hline 9 & Flicker & Never & Frequently \\
\hline 10 & Light wasted on reflector & No & High \\
\hline 11 & Buzzing & No & Yes \\
\hline 12 & Recyclable & Yes & No \\
\hline 13 & Low temperature & Compatible & Incompatible \\
\hline 14 & Starter needed & Without & Yes \\
\hline 15 & Maintenance & Low & High \\
\hline 16 & Light up time & instant-on & 3-5 seconds \\
\hline
\end{tabular}




\section{Automation System for Room Lighting Based on Fuzzy Logic Controller}

In the life of human-being, lighting is something needed during nighttimes or daytimes. However, humanbeings always give a little attention only to the operation of the lamp; they always forget to switch the lamp off as the room is empty or to decrease the number of lamps to be switched on, if the incoming light to the room is brighter. In general, the electricity consumption for the lighting will be around 25\%-50\% [8]. One of the lighting energy efficiency researches is using lighting control method. The controller will control the number of lamps to be switched on while maintaining suitable luminance for the specific condition of the room.

By the use of an automatic controller, then lighting energy consumption can be reduced. Fuzzy logic is proposed as the control method for a room lighting system with LEDs light as the controlled objects. The number of lamps depends on the condition of the room function and the standard illumination level. By using fuzzy logic controller, there are three benefits contributed, those are:

(1) Implementation of low-cost control hardware by using microcontroller based system,

(2) An automatic control system based on fuzzy logic with occupation and illumination sensing,

(3) Easiness of installation and expansion for a bigger system.

Figure 2 below clarifies the proposed system which has two sensor: the first one is occupancy sensor installed above the door, and the other one is illumination sensor butted above the windows. These sensors are connected to a microcontroller which is connected to TRIACs driver that control the number of lights fitting [8].

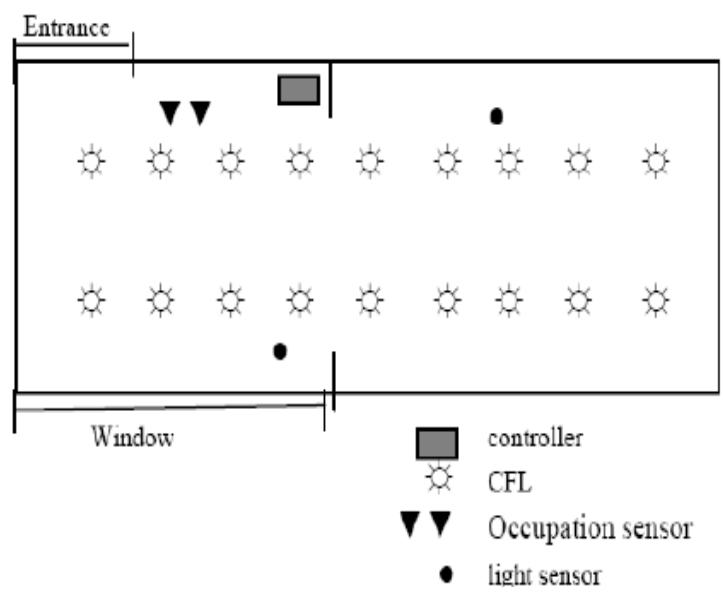

Figure 2. Room lighting based on fuzzy logic controller

\subsection{System Requirements}

This process starts by determining the illumination level of the room, measuring and calculating of room parameter, lamp position arrangement and measurement of illumination uniformity as shown in equation (1).

$$
E=\frac{\varphi+C U+L L F}{A}
$$

E: luminance (Lux), $\varphi$ : Luminance (Lumen), CU: Coefficient of Utility, A: area of illumination (m2), LLF: Light Loss Factor.

\subsection{Fuzzy Inference System}

The processes is done using fuzzy inference system (FIS) which consists of:

1) Fuzzification: is the process of mapping of crisp value to a degree of membership. The crisp values are presented by sensors.

2) Fuzzy operator (AND or OR) implementation to obtain one number that represents the result of the antecedent for that rule.

3) Implication method (min or product): the result of ifthen rule.

4) Aggregate (max or sum) of all implication result.

5) Defuzzification: process to obtain the crisp value.

The membership function used were triangular and trapezoidal function, and the method used in defuzzification was center of area (COA) as shown in equation (2).

$$
Z_{0}=\frac{\sum_{j=1}^{n} u_{Z}\left(W_{j}\right) W_{j}}{\sum u_{Z}\left(W_{j}\right)}
$$

The membership function (MF) grouping for input variables and MF for output variable are shown in Figure 3 and Figure 4. The membership functions are grouped as Very Low (VL), Low (L), Middle (M), High (H) and Very High (VH).

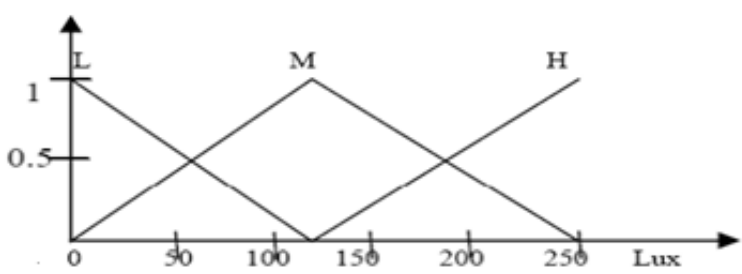

(a) MF of incoming light sensor

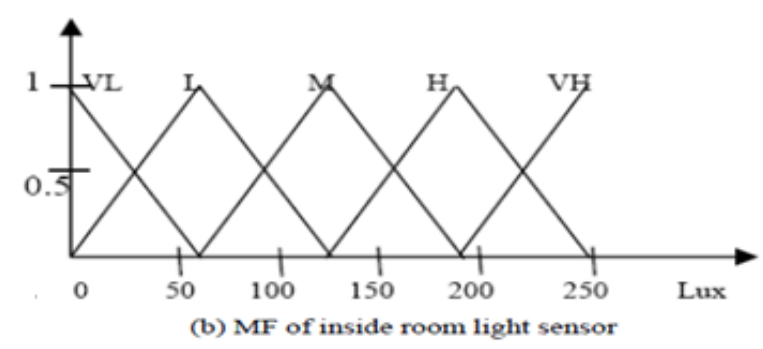

Figure 3. MF of input variable

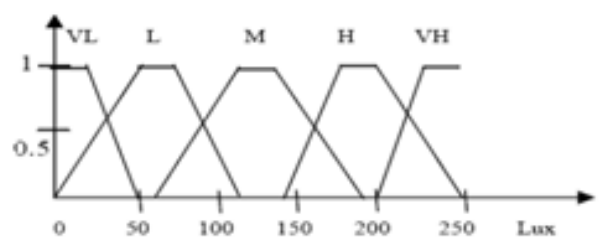

Figure 4. MF of output variable

Table 4 shows that there are 15 rules for the lighting system control, the rules are stated as follows:

$R(n)$ : IF $x_{1}$ is $A_{1} n$ and $y_{1}$ is $B_{1} n$ THEN $z$ is $Q n$

Where; 
Table 4. Fuzzy roles for lighting control system

\begin{tabular}{|c|c|c|c|c|c|}
\hline \multirow{2}{*}{$\begin{array}{c}\text { Input } \\
\mathrm{X}_{1}\end{array}$} & \multicolumn{5}{|c|}{ Input $\mathrm{y}_{1}$} \\
\cline { 2 - 6 } & $\mathrm{VL}$ & $\mathrm{L}$ & $\mathrm{M}$ & $\mathrm{H}$ & $\mathrm{VH}$ \\
\hline $\mathrm{L}$ & $\mathrm{VH}$ & $\mathrm{H}$ & $\mathrm{H}$ & $\mathrm{H}$ & $\mathrm{M}$ \\
\hline $\mathrm{M}$ & $\mathrm{H}$ & $\mathrm{M}$ & $\mathrm{M}$ & $\mathrm{D}$ & $\mathrm{L}$ \\
\hline $\mathrm{H}$ & $\mathrm{M}$ & $\mathrm{L}$ & $\mathrm{L}$ & $\mathrm{L}$ & $\mathrm{VL}$ \\
\hline
\end{tabular}

\subsection{Hardware and Software Design}

\subsubsection{Hardware Design}

The block diagram of the system hardware is shown in Figure 5. The microcontroller system receives two input signals: one is from incoming light to the room sensor and the other is from inside room sensor. These signals are put through multiplexer and analog to digital Converter (ADC) and are interfaced to the microcontroller system. The other inputs are occupation sensors, which are used to sense the people going into or out of the room [8]. The communication between the occupation sensors to the microcontroller system was accomplished through the system interrupt. The output signals of the microcontroller system are connected to the driver for controlling the switching of TRIACs in order to switch the LED on or off.

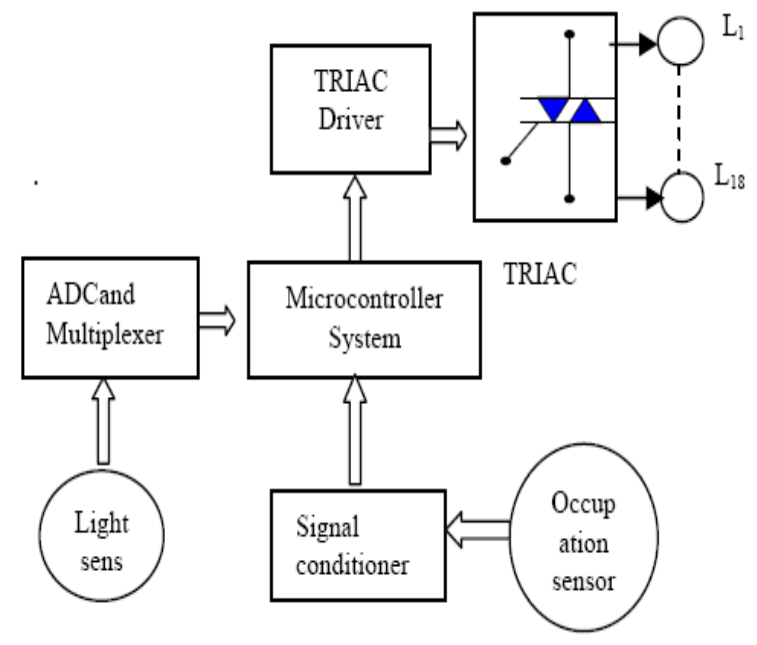

Figure 5. Fuzzy controller hardware block diagram

\subsubsection{Software Design}

The design of control system software algorithm is shown in Figure 6. The algorithm involves the following working steps:

1. Initialization of the $\mathrm{I} / \mathrm{O}$ port and system interrupt. This step is purposed to determine the I/O ports that are assigned either as input or output, also for the interruption.

2. Checking the Counter (CT), if $\mathrm{CT}=0$ then switch off all lamp; otherwise, perform sensor reading. This step is purposed to detect the occupation of the room in order to activate the lighting system once the room is occupied.

3. Fuzzification of inputs is performed where it will convert the crisp value from the luminance sensor to the fuzzy value in the software. This fuzzy value is a variable in the membership function input. The value later on will be used to decide the fuzzy value of the membership function output.

4. Application of the if-then rule is performed to select which rule(s) will be used in the computation.
5. Defuzzification is done when the value is converted to crisp value which is used as output control signal for lamps.

6. If the program stops, it exits; otherwise, it loops to check counter.

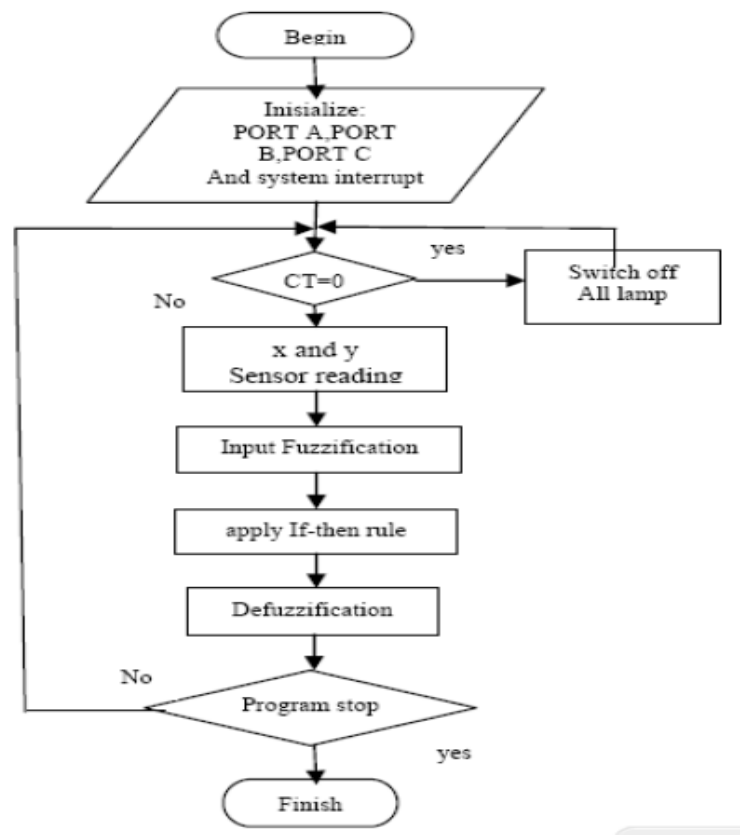

Figure 6. Fuzzy logic controller Software algorithm

Using fuzzy logic controller to control the on/off of LEDs lights which are currently used in most of buildings can save the energy around 23.9\% [8]. These results proved that the proposed controller contributes to increase energy usage efficiency. After replacing T8 fluorescent by high technology LEDs tube lamps, we will apply fuzzy logic controller to control the on/off LEDs in specific places such as showrooms, laboratories, and workshops. Selecting the location to apply automation control to light fittings will determine the saving quantity for each place. Annual energy savings is achieved upon replacing lamps from linear fluorescent lamps T8 to high technology LED lamps and installing automation fuzzy controller is $26778.56 \mathrm{kWh} /$ year.

\section{Conclusion}

Energy efficient systems provide an integral part of power saving and provide opportunities for money savings, reduction of global warming and make available saved power. The aim of this paper was to save energy by using predetermined measures and help reduce the power deficit in Gaza Strip. This paper presented a study of lighting system in Gaza Strip buildings and used Irada building at the Islamic University of Gaza as a case study. The proposed scheme required low cost investment to boost replacing T-8 FL by T- 5 and ML by HPS, medium cost to boost replacement of LED lamps instead of all FL and ML lamp program and high cost to boost the automation system for room lighting based on fuzzy logic controller at some places in the building. The proposed low cost energy saving achieved annual average energy saving of $44.7 \%$ with $18363.2 \mathrm{kWh}$ and equivalent to $\$ 2481.5$ with simple payback period of 2.8 year. The proposed medium cost 
energy saving achieved annual average energy saving around $60.4 \%$ with $24791.36 \mathrm{kWh}$ and equivalent to $\$ 3350.1$ with simple payback period of 3.7 year. The proposed high cost energy saving achieved annual average energy saving of $65.3 \%$ with $26778.6 \mathrm{kWh}$ and equivalent to $\$ 3618.7$ with simple payback period of 4.5 years. Assuming this percentage is the average saving of electrical energy that could be achieved by adopting the no cost, low cost and high cost electrical energy actions, then the rate of electrical deficit decreases by $2.8 \%$ in Gaza Strip.

\section{References}

[1] International Energy Agency (IEA), Word Energy Outlook, Middle East and North Africa Insights OECD/IEA, 2005.

[2] W.L. Lee and Hue Chen, "Benchmarking Hong Kong and China Energy Codes for Residential Buildings", Energy and Buildings, P. 1682-1636, 2008.
[3] Palestinian Energy \& Natural Resources Authority (PENRA), Accessed on: 5/3/2013, available at:

http://penra.gov.ps/index.php?option=com_content\&view=article \&id=590:2011-08-24-06-09-43\&catid=1:2009-12-29-11-0944\&Itemid $=29$.

[4] Environmental Resources International Limited (ERi). Lighting Proposal: Replacement of Existing T-8 FL Tube, Accessed on: 16/1/2013, Available at: http://www.erihk.com/lightings.htm.

[5] FL MAXiLIGHT, Energy Efficient Fluorescent Lighting: Product sheet, Accessed on: 5/3/2013 Available at: http:// www.flmaxilight.com.au.

[6] Zhonngtian Light Co., Ltd., LED Tube Light. Accessed on: 29/12/2012, Available at: http://www.ztlights.com.

[7] Alibaba Group. LED Light Replace 150W HPS. Available at: http://www.alibaba.com.

[8] Aryanto Hartoya and Seno Panjaitan, "Development of Automation System for Room Lighting Based on Fuzzy Logic Controller", International Journal of Information and Electronic Engineering, Vol. 2, No. 6, pp. 955-959, November 2012.

[9] Hatem Elaydi, Imad Ibrik, and Eyad Koudary. "Conservation and Management of Electrical Energy in Gaza Strip Using Low Cost Investment." International Journal of Engineering Research and Applications (IJERA). Vol. 2, no. 4, pp. 1152-1157, July-August 2012. 